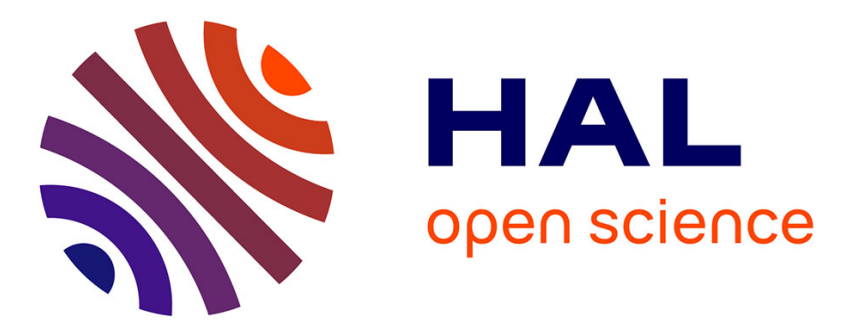

\title{
An Integrity Assessment Framework for Multi-modal Vehicle Localization
}

\author{
Arjun Balakrishnan, Sergio Alberto Rodriguez Florez, Roger Reynaud
}

\section{To cite this version:}

Arjun Balakrishnan, Sergio Alberto Rodriguez Florez, Roger Reynaud. An Integrity Assessment Framework for Multi-modal Vehicle Localization. 2019 IEEE Intelligent Transportation Systems Conference - ITSC, Oct 2019, Auckland, New Zealand. pp.2976-2983, 10.1109/ITSC.2019.8916874 . hal02393213

\section{HAL Id: hal-02393213 \\ https://hal.science/hal-02393213}

Submitted on 4 Dec 2019

HAL is a multi-disciplinary open access archive for the deposit and dissemination of scientific research documents, whether they are published or not. The documents may come from teaching and research institutions in France or abroad, or from public or private research centers.
L'archive ouverte pluridisciplinaire HAL, est destinée au dépôt et à la diffusion de documents scientifiques de niveau recherche, publiés ou non, émanant des établissements d'enseignement et de recherche français ou étrangers, des laboratoires publics ou privés. 


\title{
An Integrity Assessment Framework for Multi-modal Vehicle Localization
}

\author{
Arjun Balakrishnan ${ }^{1,2}$, Sergio A. Rodríguez F. ${ }^{1,2}$ and Roger Reynaud ${ }^{1,2}$ \\ ${ }^{1}$ ENS Paris-Saclay. Pari-Sud University. Paris-Saclay University, 91405 Orsay, France \\ ${ }^{2}$ SATIE Laboratory - UMR 8029 CNRS joint research unit
}

\begin{abstract}
Integrity monitoring of sources and process associated with localization systems used in autonomous navigation applications is of paramount importance. In this work, we present a novel source-level integrity monitoring method with a focus on generalization, scalability and versatility. A crossconsistency assessment based framework is developed to monitor the integrity of different spatial data sources. The proposed method is applied to several datasets, which demonstrates promising behavior of our integrity markers in identifying and quantifying unreliable data from sources. Several scenarios, which are normal occurrences in challenging driving conditions, are considered in the experiments and the advantages of the proposed integrity monitoring process are highlighted.
\end{abstract}

Index Terms-Integrity assessment, intelligent vehicles, localization

\section{INTRODUCTION}

The era of intelligent and autonomous driving systems is waiting at the threshold to transform the history of transportation methods. The general consensus among the researchers and industries in this area is optimistic that the deployment of such systems could drastically improve the safety and efficiency of driving compared to humans. While sensorfusion based autonomous driving systems are rapidly maturing to be present even in commercial vehicles, the reliability concerns of this process are largely overlooked. One of the main advantage of humans over autonomous driving systems is the ability to monitor numerous parameters from driving scenarios to understand the context before making decisions.

For example, a driver can decide not to drive or drive slowly under poor visibility conditions. Likewise, a driver can choose to follow signboards and other visual information for reliable driving in case he/she observes inconsistent GPS localization in an urban canyon or an erroneous map while driving in the countryside. It is easy to note that the knowledge of context and the integrity associated to every data source, modules and processes involved in autonomous driving systems, is crucial for replacing humans in the driving process. Most of the advancements in this area address the task of integrity monitoring explicitly by introducing redundancy in sensors, using sensors with advanced features, monitoring repetitive journeys, or by assuming one source (often high-quality digital maps) as reliable ground truth. In this paper, we approach the integrity monitoring of sources in a more generalized and fundamental way to aid the localization process.

\section{SELF-ASSESSMENT}

The term "integrity" has different definitions in different scientific areas. In the case of intelligent transport systems, integrity is the measure of trust that can be placed in the correctness of the information supplied by the total system. This also includes the ability of a system to provide timely and valid warnings to the user when the system must not be used for the intended operation [1]. Majority of the scientific literature reviewed in this work extends this definition to the contexts of localization, sensor-fusion and autonomous navigation. They primarily focus on monitoring the integrity of different processes in localization and provide alerts and warnings. Works like [2] and [3] are intended to monitor the integrity and detect the faults in localization for critical applications, like alerting drivers or maintaining Geo-fencing. Fault Detection and Isolation (FDI) schemes are used for this purpose, which generates robust models for the residuals between different sources and uses them in the decision making process that identifies faults[4].

Since this work focuses on integrity monitoring at the source level before using the data sources for the localization process, we limit our self-assessment to data source specific integrity monitoring techniques. In [2], Receiver Autonomous Integrity Monitoring (RAIM) algorithm is used to detect faults with redundant GPS pseudo-range measurements. Two GNSS sensors - one used for map matching and another combined with proprioceptive sensors for vehicle position estimation - are used in [5] to add data redundancy in fault detection in GPS localizations. In [6], inertial sensor is coupled with GPS sensor, so that faults due to drift in inertial data and bias in GPS data can be identified simultaneously using a Generalized Likelihood Test (GLT). Evidently, these approaches require one or more of these options - i) GPS sensors with specific capabilities [7] (raw measurements of carrier phase of GPS signals), ii) multiple GPS sensors, iii) complimentary sensors - to achieve the integrity monitoring of GPS data. As far as the integrity of navigation map data is concerned, in [8] an enhanced RAIM algorithm is proposed which can also incorporate a map-matching process for integrity monitoring. Authors of [5] extended their work to integrity monitoring of navigation maps by employing a sequential Fault Detection, Isolation and Adaptation (FDIA) method on localization estimates from repetitive journeys through the same route [9]. To the best of our knowledge, there are few integrity monitoring techniques for other sensors that can be used in vehicle 
localization, such as vision systems, range sensors, LIDAR systems etc.

In the interest of establishing the foundation of our novel approach, the definition and structure of "integrity" is adapted using the concepts from data sciences. This adaptation is justified, as this work considers physical sensors as data sources represented in common reference frame and dimensionality. According to [10], integrity is the measure of overall accuracy and consistency of data. Accuracy refers to the correctness of validated data from a source. When valid data from different sources match each other, data consistency is achieved and sources are said to be reliable. Further improving upon this structure, our method tries to detect the accuracy of each source and monitor the consistency between them to provide quantitative markers for their integrity.

\section{Methodology}

While proprioceptive sensors measure the state of the vehicle, most of the exteroceptive sensors provide spatial information such as global positioning of the vehicles, distance to obstacles and structure of the environment. Modern intelligent driving systems incorporate 3D and 2D digital maps as a data source to plan the navigation as well as improve the localization of the vehicle. As the number of sensors and data sources increases, it is important that their confidence should be estimated at the sourcing level so that the localization process that uses those sources produces consistent output. If a source is found out to be less reliable, its effect can be reduced while estimating the localization of the vehicle. In general, providing a localization estimate along with its integrity estimate can greatly benefit the safety of the vehicle.

In this paper, we propose an intuitive and novel approach to address this problem. The generalized functional block diagram of this approach shown in Fig. 1, is inspired from [9]. Consider $\mathrm{N}$ data sources which can continuously provide spatial information relevant to the localization of the vehicle in the driving scenario. As a prepossessing step, outliers are removed from each source according to the prior knowledge about their models and data acquisition technique. Then all data are transformed to a chosen reference frame using different geometric transformations based on their original reference frame, scale and units. A common model is applied to each of the data sources represented in the chosen reference frame and these models are compared using a crossconsistency analysis routine which in turn provides integrity markers for each source.

For the development and analysis of the proposed concept, a multi-modal system with three different data sources is used in this work. This section describes the approach to sensor integrity assessment in which digital maps are considered as a data source along with GPS receiver and cameras. GPS coordinates, detected lane markings from vision and data from digital maps are considered in a common framework and their models are extracted. Quadratic polynomials are used as models, assuming road structures and driving tracks can be

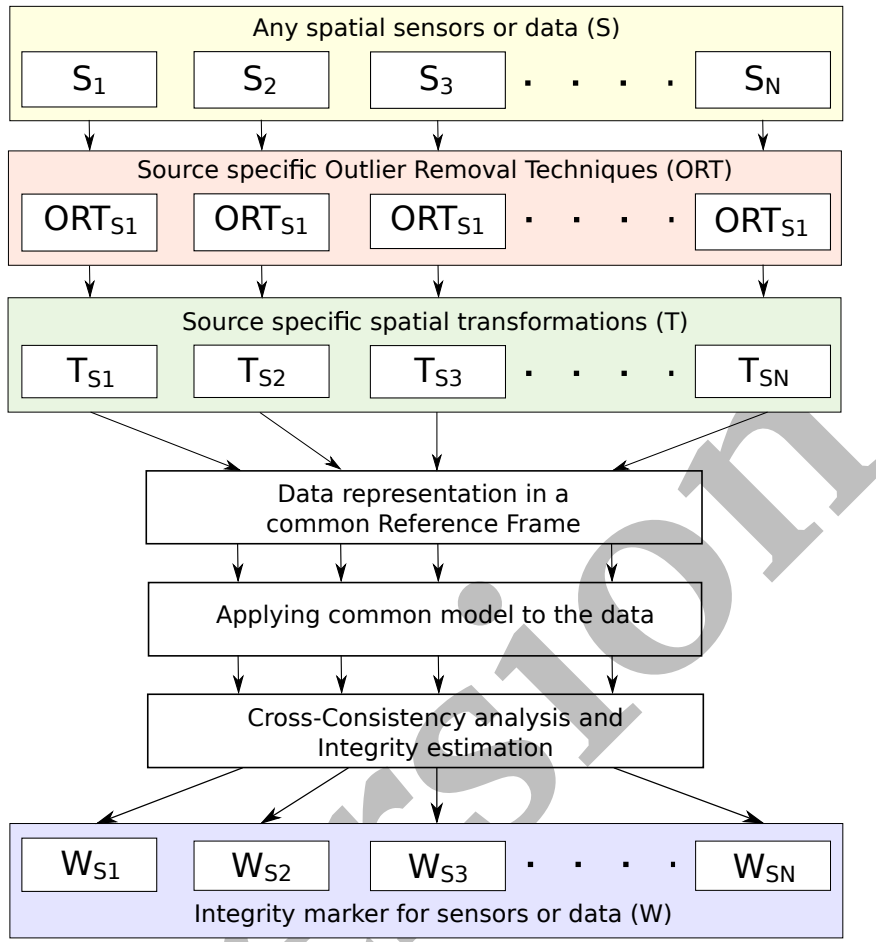

Fig. 1: Functional block diagram of the methodology proposed inspired from [9]. Sec. III-A outlines the pipeline of the procedure: Sec. III-B details the outlier removal techniques and Sec. III-C describes the transformations used. Sec. III-D defines the cross-consistency analysis followed by the definition of integrity markers in Sec. III-E

approximated locally using single convex functions [11]. In order to robustly estimate the models, M-estimators are used and outliers are removed from each source. Obtained models from each data sources are then compared by estimating their fit across other data sources. This enables a cross-consistency analysis of each data sources, thus providing an effective and quantifiable marker to represent their reliability.

\section{A. Data Handling Strategy}

Due to the importance of accurate data representation in the proposed method, this section is dedicated to describe the evolution of data from each source considered in this paper. GPS sensors provide data in the form of (latitude, longitude) which are in degrees of angles in International Terrestrial Reference Frame (ITRF). Large scale digital maps - especially OpenStreetMap (OSM) used in this work - are also constructed using Geo-localized nodes expressed as coordinates in ITRF, making GPS data and map data coherent. Since ITRF is a spherical coordinate system, projection to a Cartesian coordinate system is required to directly apply analytic geometric functions and operations. Mercator projection is used to convert GPS and map data to EastNorth-UP (ENU) frame, which is a Cartesian coordinate system with real-world distance units.

Assuming the vehicle's body frame is close enough to the camera frame, principal point of the camera and principal 
axis are taken as the origin and $\mathrm{X}$-axis of the ego-frame of the vehicle. By using the parameters of calibrated camera, Inverse Perspective Mapping (IPM) can provide Bird-EyeView (BEV) in which road plane is reconstructed. Using a lane detection algorithm, lane markings are detected in BEV and reprojected as a set of real-world spatial points in camera frame. Each lane markings are detected as blobs and their Yaxis offsets are removed and aligned to $\mathrm{X}$-axis, which allows us to treat left and right lane markings as a single set of data points.

The ego-frame of the vehicle is used as the common reference frame for the considered scenario, hence lane markings from vision do not need any transformation. Assuming GPS sensor is placed near to vision system, the current GPS position will be the same as the origin of the ego-frame. In order to detect and remove outliers and to reliably fit the model, history of GPS points of a predefined length (20) is considered. Geographical nodes belonging to the road in which vehicle is traveling, are extracted from map source. At this point, the data sources considered in the work are denoted as follows.

$G_{\text {enu }}$ : set of current and $\mathrm{n}$ previous GPS position fixes in ENU-frame

$M_{\text {enu }}$ : set of locations of nodes corresponding to the road segment in which vehicle is traveling, in ENU-frame

$V_{\text {ego }}$ : set of detected lane marking pixels reprojected in egoframe

To proceed further, $G_{\text {enu }}$ and $M_{\text {enu }}$ need to be transformed to ego-frame. Since current GPS coordinate is at the origin of ego-frame, $G_{e n u}$ and $M_{\text {enu }}$ can be translated using current GPS coordinate, thus this transformation is reduced to rotation using the estimated orientation of the vehicle. In theory, orientation estimation can be done by calculating the angle between the current and previous GPS points. But noisy GPS data does not allow this approach. Mean of the angles between current and few immediate previous points could be used to reliably estimate the orientation of the vehicle. Since outliers can heavily affect this approach, outlier removal should be done prior to this process. Outlier removal and angle estimation (hence the transformation) are explained in detail in following sections. Finally, GPS data points and nodes from map are rotated using the estimated angle.

\section{B. Outlier removal}

According to the characteristics of outliers present in each data source, different outlier removal techniques are necessary. Lane detection algorithm can have incorrectly detected markings as outliers, which may not follow the orientation or width of the typical lane markings in ego frame. These constraints are used for outlier removal from vision data. For each blob detected, image moments are calculated to estimate the orientation and size of each blob. A range for orientations in which most number of long lane markings are present, is estimated. Any detected marking that does not fall in this orientation range is further examined for its size. If

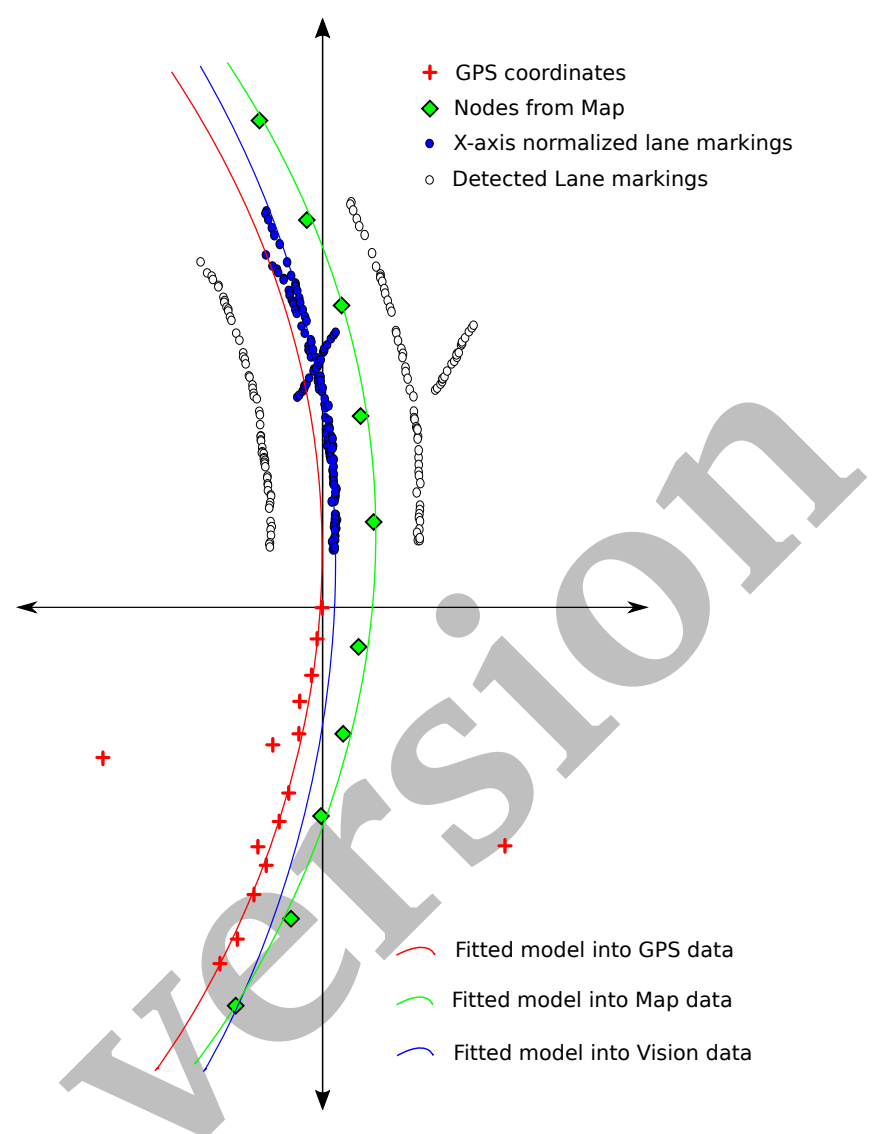

Fig. 2: Representation of data from different sources in egoframe during typical scenario

the length is short or the width is bigger than expected width of lane markings, they are detected as outliers. Blobs outside this orientation range, that are similar to long lane markings will be passed as inlier. This can be seen as an example of erroneous visual data.

$G_{\text {enu }}$ and $M_{\text {enu }}$ have similar outlier characteristics. Outliers in these are the coordinates which do not fit to the characteristic model of them. Least squares method is used to fit quadratic polynomials of the form $y=p(x)=a x^{2}+b x+c$ to $G_{\text {enu }}$ and $M_{\text {enu }}$. Once the models are extracted ( $p_{g}$ and $p_{m}$ for $G_{e n u}$ and $M_{\text {enu }}$ respectively), the residual distribution of each data source are computed $\left(r_{g}, r_{m}\right)$. To model the distribution of residuals, mean and standard deviation are computed. Using these as initial estimates, Huber's robust parameter estimation method is employed to get the final model of the distribution. Outliers are detected and removed using $2 \sigma$ threshold on $r_{g}$ and $r_{m}$.

\section{Transformations}

Let $G_{\text {enu }}^{\prime}, M_{\text {enu }}^{\prime}, V_{\text {ego }}^{\prime}$ be the outlier removed dataset. As discussed, to represent $G_{e n u}^{\prime}$ and $M_{e n u}^{\prime}$ in ego-frame of the vehicles, orientation of the vehicle needs to be estimated. To do this, $G_{e n u}^{\prime}$ and $V_{e g o}^{\prime}$ are used because of two reasons. Firstly, after translating $G_{\text {enu }}^{\prime}$ to the current GPS coordinate, their origins are the same. We can not assure that there are map nodes at the current vehicle locations in this same 
analogy, hence map data can not be used for angle estimation. Secondly, in a chronological point of view, a set of previous GPS coordinates represents the history of the vehicle's trail and detected lane markings represents the prediction of the trail. Intuitively, these two demand consistency under all normal driving scenarios.

At this point, we introduce the operation RFS (Rotate-FitSample), which is defined as

$$
P_{D \theta}:=\left\{\left(x_{1}, y_{k 1}\right),\left(x_{2}, y_{k 2}\right), \ldots,\left(x_{N}, y_{k N}\right)\right\}=\operatorname{RFS}(D, \theta, N)
$$

where $D$ is the set of data points from a source represented in ego-frame. $R F S$ rotates $D$ about the origin by angle $\theta$, then robustly fits second degree polynomial to the result and sample the polynomial into $N$ 2D points equidistantly along $\mathrm{X}$-axis.

At the starting of the transformation process from ENUframe to ego-frame, $G_{\text {епи }}^{\prime}$ and $M_{\text {eпи }}^{\prime}$ are translated to the origin of ego-frame by subtracting the current GPS coordinates. For the sake of simplicity, the notations for these translated data points are kept the same. The initial estimate of orientation of the vehicle $\left(\theta^{\prime}\right)$, is the mean of the angles between current and 5 previous GPS positions from $G_{e n u}^{\prime}$ (outlier removed GPS data). $R F S\left(G_{\text {enu }}^{\prime}, \theta^{\prime}, 20\right)$ and $R F S\left(V_{\text {ego }}^{\prime}, 0,20\right)$ gives the initial framework for angle estimation method. The angle is found out by iterative minimization of the Euclidean distance between these two set of points.

$$
\hat{\theta}=\underset{\theta}{\arg \min }\left(\left\|P_{G \theta}-P_{V 0}\right\|_{2}\right)
$$

$G_{e n u}^{\prime}$ and $M_{e n u}^{\prime}$ are rotated using $\hat{\theta}$ to get $G_{e g o}^{\prime}$ and $M_{\text {ego }}^{\prime}$. The final representation of all the data from different sources as shown in Fig. 2 .

\section{Cross-Consistency estimation}

In this section, we propose a method to analyze the consistency between different data sources. If the data from each source is accurate, their models should exhibit similar properties such as similar curvature and tangent angles. After the representation of data in ego-frame, quadratic polynomial models $p_{g g}, p_{m m}$ and $p_{v v}$ are estimated from $G_{e g o}^{\prime}, M_{\text {ego }}^{\prime}$ and $V_{\text {ego }}^{\prime}$ respectively, as shown in Fig. 2. Next step is to assess the 'Goodness-of-Fit' (GoF) of each of these polynomial models across all possible data sources. The characteristics of a quadratic polynomial are defined by its second and first order parameters and the constant parameter represents the Y-axis offset of the polynomial at the origin. Hence, to check the GoF of a model to a different set of data points, it can be shifted to the location of those data points. This is achieved by changing the constant parameter of the model under consideration, by the same of the model of the data source to be considered. Hence three data sources considered in our proposed method will provide 9 combinations as follows.

$$
\begin{array}{rrr}
p_{g g}:\left(g_{a}, g_{b}, g_{c}\right) & p_{v g}:\left(v_{a}, v_{b}, g_{c}\right) & p_{m g}:\left(m_{a}, m_{b}, g_{c}\right) \\
p_{g m}:\left(g_{a}, g_{b}, m_{c}\right) & p_{m m}:\left(m_{a}, m_{b}, m_{c}\right) & p_{v m}:\left(v_{a}, v_{b}, m_{c}\right) \\
p_{g v}:\left(g_{a}, g_{b}, v_{c}\right) & p_{m v}:\left(m_{a}, m_{b}, v_{c}\right) & p_{v v}:\left(v_{a}, v_{b}, v_{c}\right)
\end{array}
$$

Where $P_{i j}$ represents the polynomial model used to analyze GoF of $P_{i i}$ to $j_{\text {ego }}^{\prime}$ data source. GoF of each of these models are defined based on normalized sum of weighted residuals. In order to weight the residuals, a measure of curvature is computed for each point on the polynomial function using in Eq. 3

$$
c_{i}=\frac{2 a}{\left(4 a^{2} x_{i}^{2}+4 a b x_{i}+b^{2}+1\right)^{\frac{3}{2}}}
$$

Where $a, b$ are polynomial model parameters and $x_{i}$ is the $\mathrm{x}$-axis value of the point at with curvature needed to be estimated.

Weighing residuals using measure of the curvature has particular significance. The curvature of the model is most significant and observable at smaller values along the $\mathrm{x}$-axis. Likewise, while estimating a polynomial model from a set of data points, points that are near origin will determine the curvature of the model more than the farther ones. This property intrinsically incorporates more importance for the data points near the vehicles current position, which are preferred for cross-consistency analysis. On the other hand, if the vehicle is on a straight road segment, none of the sources will provide a model with a considerable curvature (i.e, close to zero value). Hence incorporating the measure of the curvature into the GoF estimation will automatically compensate for the limitations of this approach. Finally, we define the marker for cross-consistency analysis as

$$
e_{i j}=\frac{1}{N_{j}} \sum_{l=1}^{N_{j}}\left(c_{l}\left(y_{l}-p_{i j}\left(x_{l}\right)\right)\right) \quad \forall\left(x_{l}, y_{l}\right) \in j_{\text {ego }}^{\prime}
$$

where $e_{i j}$ is GoF marker associated with $P_{i j}$ and $N_{j}$ is the total number of data points in $j_{\text {ego }}^{\prime}$. All possible $e_{i j}$ values are arranged in an evolving square matrix $E$ as given below.

$$
E=\left[\begin{array}{lll}
e_{g g} & e_{m g} & e_{v g} \\
e_{g m} & e_{m m} & e_{v m} \\
e_{g v} & e_{m v} & e_{v v}
\end{array}\right]
$$

\section{E. Integrity Markers}

In section III-D, we formulated a quantifiable marker to express the consistency of each source with the other sources. At this point, if $e_{i j}$ is a low value, it can be inferred that $i_{\text {ego }}^{\prime}$ is consistent with $j_{\text {ego }}^{\prime}$. In the case of $i=j$, a low $e_{i j}$ value will mean that model estimation is reliable. However, to estimate the integrity of a source, we have to combine these notions in a sensible way. The idea is to provide a weighing parameter for each source, which can be easily incorporated into sensor-fusion or localization improvement algorithms used in autonomous driving process. Combining the GoF of a polynomial model to its own source and the GoF to other sources will represent the consistency of that model, hence the integrity of the respective data source. Hence the integrity markers are defined as below.

$$
W_{i}=1-\frac{\sum_{\forall j} e_{i j}}{\sum_{\forall i, j} e_{i j}}
$$


These weights, $W_{g}, W_{m}$ and $W_{v}$, represents the integrity of data obtained from GPS, map and vision respectively. Higher values of these weights correspond to higher integrity of their respective sources and vice versa. The formulation of this marker tells us how much of the total error observed in the consistency analysis is caused by each data source.

\section{F. Fault and Feasibility Predictors}

The cross-consistency estimation process assumes that the models for all data sources are always present and their consistency is an observable quality at all the instances. There can be several scenarios where these assumptions could not be held true. In this section, we try to address such scenarios with Fault and Feasibility Predictors $(F P)$ defined for each of them.

- $F P_{g}$ : Fault Predictor for GPS - If GPS fix is not available or the current GPS fix is an outlier at any given time. Under these cases, we will use the previous reliable GPS localization and proceed with the process, but to account the unreliability of the process $F P_{g}$ is raised.

- $F P_{m}$ : Feasibility Predictor for Map - If there are two or fewer nodes in the map cache, a reliable fitting of the polynomial model is not possible, thus raising $F P_{m}$. This happens when road sections do not have a considerable curvature because cartographers tend to use fewer nodes for constructing maps in such cases.

- $F P_{v}$ : Fault prediction for Vision - If there are no long and continuous lane markings detected. Under certain challenging situations, characteristic lane markings may not be present or may not be detected. The model fitting may work on detected noises in visual data, but its validity could be seriously affected. $F P_{v}$ addresses these conditions.

- $F P_{S}$ : Feasibility predictor based on speed - If the vehicle comes to a stop or moving significantly slow, the history of GPS fixes used to extract the model from GPS source could not represent the situation. Hence if the GPS history does not span over a fixed threshold length, $F P_{S}$ is raised.

- $F P_{t}$ : Feasibility predictor based on turning - If a vehicle is taking a hard turn from one road to another discontinuous road, $F P_{t}$ is raised. In these scenarios, there need not be any consistency between what vehicle observes in front and the history of vehicle localization. $F P_{t}$ will be kept raised until the history of vehicle localization completely belongs to the new road.

When any of these FPs are raised, the output of the crossconsistency analysis should be taken with respective unreliability expected. Though FPs need some hard thresholds to be defined, they need not be highly empirical and can be easily derived using pragmatic understanding of the situations.

\section{EXPERIMENTS AND DISCUSSION}

Experiments are conducted with datasets available in KITTI benchmark suit [12] to establish proof-of-concept. RTK GPS

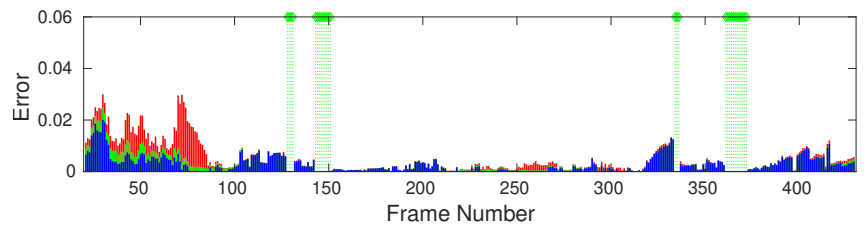

Fig. 3: Error evolution for Dataset 1. Red: GPS error, Green: Map error, Blue: Vision error, Green dotted lines: $F P_{m}$

fixes available in these datasets are modified with additive white noise to emulate poor localization fixes as well as random outliers provided by generic GPS sensors. This section considers the use of four scenarios to test proposed method. The result of integrity assessment is reported as three scores at each time-step (in this case frame number, since GPS and visual data are sampled synchronously) : total error observed $\left(E_{t}\right)$, ratio of errors due to each data source in the total error observed $\left(R=E_{g}: E_{m}: E_{v}\right.$, such that each are in normalized range $(0,1)$ and $\left.E_{g}+E_{m}+E_{v}=1\right)$ and reliability of integrity assessment $(F P) . F P$ encloses the set of all fault detection and feasibility criteria for integrity assessment discussed in section III-F. The following discussion on results is structured in a specific format. Sections with high total error observed are identified from each dataset and a representative scenario from each section is examined to explain the cause and confirm the validity of the contribution by each data source to the total error.

\section{A. Dataset 1 - 2011_09_26_drive_0028}

This dataset contains images that are polluted with multiples shadows from surroundings which reduces the performance of lane marking detection. Evolution of errors observed in the integrity estimation process on this dataset is given in Fig. 3 . Despite the low error observed throughout this dataset, it is interesting to notice the contribution of different sources in the total error in several conditions. In the scenario at frame number 28, a larger curvature is observed in vision due to the entry of vehicle into a curve from a straight road segment, which results in the ratio of errors 0.191:0.183:0.625. At frame number 77 , the vehicle exits the same curve to a straight road segment, causing higher curvature in GPS which results in the ratio 0.883:0.108:0.011. Two or less nodes are available from the maps at all the straight road scenarios marked with green dotted lines $\left(F P_{m}\right)$ in Fig. 3

\section{B. Dataset 2 - 2011_09_26_drive_0029}

In dataset 2, a compound and a reverse curve follows a left turn from a red light stop at the end of straight road segment. Four types of $F P$ markers are observed in this process. In the straight line segment, few $F P_{v}$ and $F P_{m}$ are observed due to lack of information. As the vehicle switches the lanes to the left most lane for the turn, an increase in $E_{t}$ is observed due to poor model fitting on GPS data until the $F P_{s}$ is produced from frame number 186 to 265. In Fig. 5a a situation during the stop is shown. Note that the model is ill-fitted on all the data sources. 


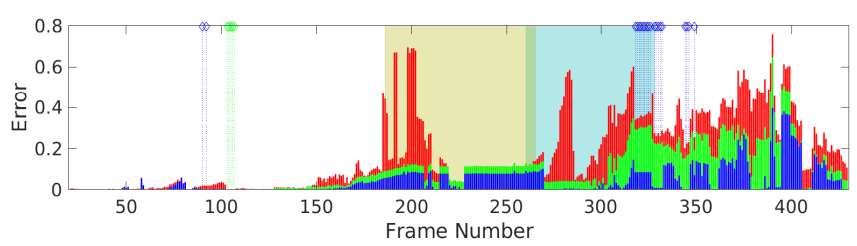

Fig. 4: Error evolution for Dataset 2. Red: GPS error, Green: Map error, Blue: Vision error, Green dotted lines: $F P_{m}$, Blue dotted lines: $F P_{v}$, Light brown: $F P_{s}$, Light blue: $F P_{t}$

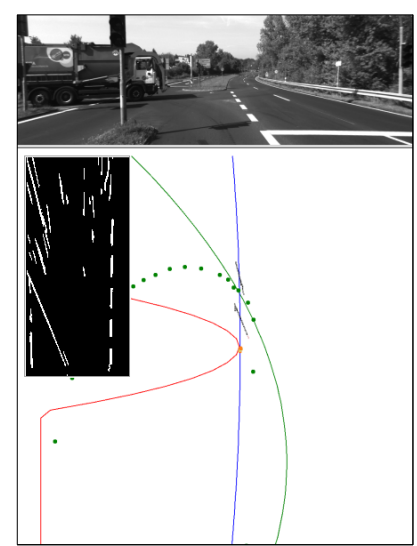

(a) Scenario at frame number 207
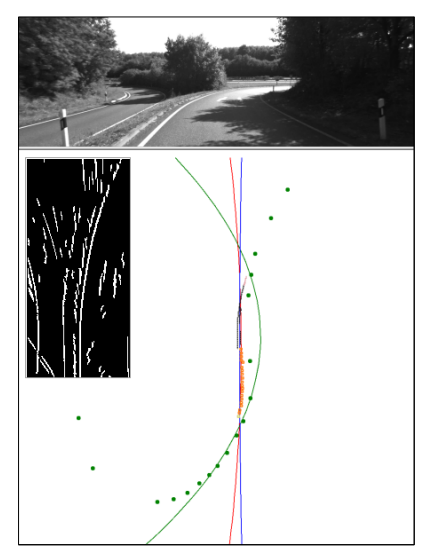

(b) Scenario at frame number 390
Fig. 5: Different scenarios from dataset 2

GPS points are concentrated around vehicle's stop location, causing unstable polynomial models. While vision observes the straight road ahead, moving vehicles present in front of the vehicle which cause faulty lane marking detection, thus unstable polynomial models. $F P_{s}$ encompasses all these unreliability in the integrity assessment process until the vehicle starts to move again from 265. But from 259 onward $F P_{t}$ is produced indicating the hard turn that results in wrong lane detection and inconsistent GPS history. The turning section is then followed by vision data outages due to challenging light conditions and shadows present along the curves (similar to the situation shown in Fig. 5b). Integrity assessment works well on compound curve section due to highly stable curve models estimated. Even though $E_{t}$ is high, combined with the $R_{e}$, integrity assessment gives behavior of each data source accurately.Fig. 5b shows the limitation of the proposed method due to the presence of a reverse curve. The curve vehicle had traveled and the curve vehicle observes ahead are in opposite direction in this situation and a second degree polynomial fails to model the map data. This gives the peak $E_{t}$ at frame number 390 (the inversion point of the reverse curve) and a ratio of 0.147:0.329:0.524, where this indicates vision is the most unreliable source since other two models are coherent following the same direction.

\section{Dataset 3 - 2011_09_26_drive_0042}

Only a part of this dataset (from frame number 400) is shown as result in Fig. 6, because of the long straight highway at the beginning of the dataset. $F P_{v}$ and $F P_{m}$ are observed in

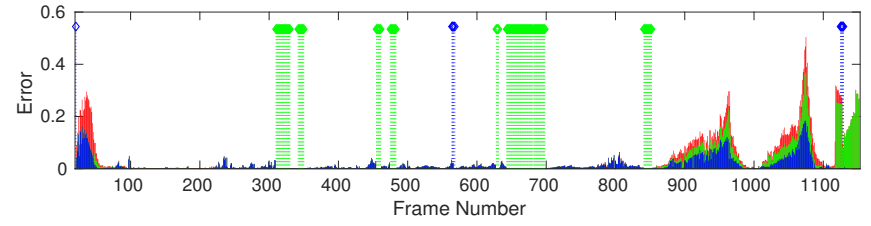

Fig. 6: Error evolution for Dataset 3. Red: GPS error, Green: Map error, Blue: Vision error, Green dotted lines: $F P_{m}$, Blue dotted lines: $F P_{v}$

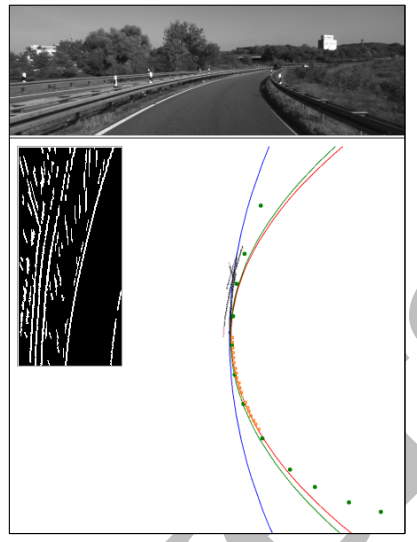

(a) Scenario at frame number 962

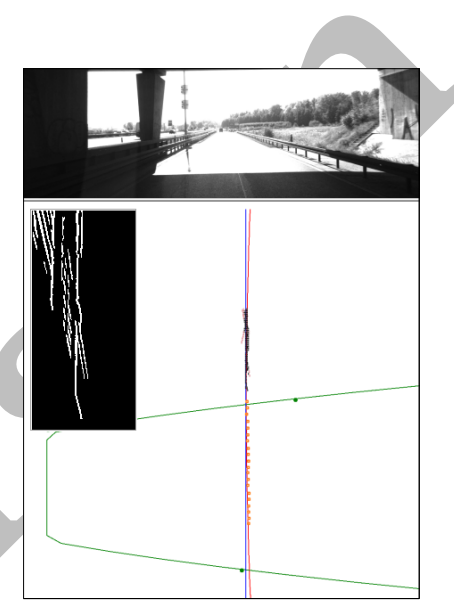

(b) Scenario at frame number 1140
Fig. 7: Different scenarios from dataset 3

this section and most of the error in this section is from vision due to challenging light conditions and presence of markings from road splits and road merges. After Frame number 890 , the vehicle enters a continuous curved road split where increase in $E_{t}$ is observed. As evident from Fig. 6 majority of the error is observed in vision data due to the limitation of IPM transformation used to create BEV images for lane detection. Since these are large radius curves, the IPM transformation fails to map the curvature of the road farther from the vehicle position. A typical example of this is shown in Fig. 7a. After frame number 1117, an another section of high $E_{t}$ is observed with majority of which is due to $E_{m}$. The vehicle is approaching a overpass with multiple lanes, which caused an erroneous map matching result. The integrity assessment method manages to identify this because of the inconsistent model obtained from the map data. It is also worth noting that few $F P_{v}$ s are also detected in this section when the vehicle was completely under the shadow of the overpass where no lane markings were visible.

\section{Dataset 4 - 2011_09_26_drive_0070}

In dataset 4 , vehicle enters a straight main road from a curved side road. Until the vehicle is completely entered on the main road, $F P_{t}$ is produced, which helps to avoid the inexplicable errors observed from each source. But from Frame number 35, a high $E_{g}$ is observed until frame number 50. In this scenario, GPS history is not along the path in which vehicle is currently moving while vision and map data are consistent. It can be observed that $E_{g}$ is continuously decreasing as the 


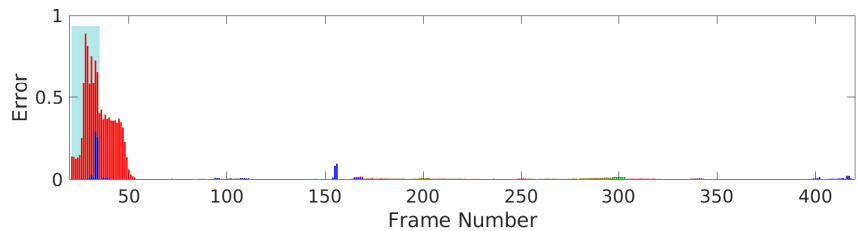

Fig. 8: Error evolution for Dataset 4. Red: GPS error, Green: Map error, Blue: Vision error, Light blue: $F P_{t}$

GPS history increasingly represent the actual road on which vehicle is moving.

\section{E. Performance Evaluation}

In order to assess the performance of the proposed integrity assessment method, errors and faults are introduced to each source under controlled conditions to characterize the behavior of the proposed method. To simulate errors in GPS, outliers are introduced whereas random road sections are selected from the map to simulate erroneous map inputs. Obtained vision markings are rotated by randomly generated angles, to serve as instances of poor detections from vision systems. Errors introduced in GPS are measured as the distance between ground-truth GPS position and the generated noisy GPS position. The angle between the actual road section and randomly selected road sections characterizes the errors introduced in the map. The angle used to rotate all lane marking detections provides a measure of error introduced in vision data. In Table I, an example of performance evaluation on Dataset 1 is shown. The above mentioned errors are systematically applied on certain intervals in the dataset. Total error $E_{t}^{*}$ and the ratio of errors $R^{*}$ obtained from the modified dataset are compared to that of the original dataset ( $E_{t}$ and $R$ respectively). Not only we observe higher $E_{t}$ in each case, but also the increment observed is correlated to the amount of error introduced. The same can be stated for $R$ as well. At frame number 50, GPS error introduced is of $1.684 \mathrm{~m}$, which constitutes an increment of 0.0071 in $E_{t}$ and changes $R$ from 0.511:0.175:0.314 to 0.628:0.130:0.242. But for an introduced GPS error of $9.862 \mathrm{~m}$ at frame number $350, E_{t}$ has increased 0.2852 and a distinctive change in $R$ from 0.122:0.048:0.830 to 0.965:0.008:0.026 is observed. Similar associations can be observed for other sources and the behavior can be explained using the measure of the errors introduced.

In the next experiment, GPS source is focused to establish the performance of the proposed method on two different GPS localization scenarios. Two sections of errors are introduced in Dataset 1: i) continuous outliers for a trajectory length (30 fixes) more than the length of GPS history used in this work (i.e, 20 fixes) and ii) a continuous added lateral offset (length: 30 frames) to actual GPS localization fixes. The former can simulate the behavior of GPS in a tunnel, whereas the latter can be approximated as the behavior of GPS in an urban canyon due to multi-path effects. The result of this experiment is shown in Fig. 9. Frame numbers from 200 to 230 contain introduced outliers and depending on the

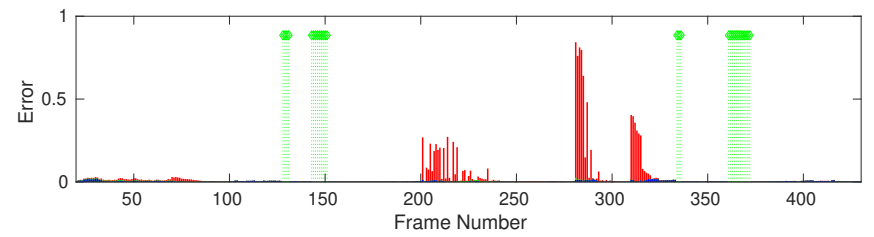

Fig. 9: Performance evaluation on Dataset 1. Red: GPS error, Green: Map error, Blue: Vision error, Green dotted lines: $F P_{m}$

magnitude of them, the process immediately responded and higher $E_{t}$ and higher ratio for GPS in $R$ is observed. But when offset is introduced between frame number 280 and 310 , two distinct high $E_{t}$ sections are observed instead of one. When the offsets lasts more than the length of GPS history used, entire GPS history used for the process can contain only measurements with offsets. Since this method uses curvature consistency between models and discards consistency in offsets, such cases may observe good crossconsistency thus providing lower $E_{t}$. After frame number 290, we can observe the decrease in $E_{t}$ due to the fact that more than half of the points in GPS history are with offset, hence resulting in a consistent model to other sources. The opposite is observed once the offset is removed at frame number 310 . Until frame number 320 , the model estimated corresponds to the GPS points with offset present in the history which leads to high $E_{t}$ values. This is a limitation attributed to the proposed method. This can be avoided by using an offset tracking mechanism between data sources.

\section{Conclusions}

In this work, a novel approach to the integrity assessment of data sources used in autonomous navigation is proposed and proof-of-concept is provided. The method is adaptable to a variety of sensor modalities and data sources that can provide spatial information about the surroundings of a vehicle. It also does not depend on the choices of preprocessing methods, detection algorithms or data association methods. There are only two requirements to apply this integrity assessment method to any sensor combinations and situations: i) possibility of representing all considered data sources in a common frame and ii) Formulating a model that could be used to represent all individual data sources in the chosen common frame.

In our experiments, vision data from a camera, localization fixes from a GPS sensor and geographical information from a digital map are used as data sources. A second-degree polynomial is used as the model for all the data sources. By conducting experiments on different standard datasets, it has been shown that the proposed method could provide a reliable integrity marker for each data source by exploiting the cross-consistency between them. Integrity marker for vision data can account for the effect of quality of lane markings detected, erroneous detection, lack of information and challenging lighting conditions. Integrity marker for GPS data monitors the quality of localization fixes and its 


\begin{tabular}{|c|c|c|c|c|c|c|c|}
\hline Frame no. & GPS error $(\mathrm{m})$ & Map error $(\mathrm{rad})$ & Vision error $(\mathrm{rad})$ & $E_{t}$ & $E_{t}^{*}$ & $R$ & $R^{*}$ \\
\hline \hline 50 & 1.684 & 0 & 0 & 0.0217 & 0.0288 & $0.511: 0.175: 0.314$ & $0.628: 0.130: 0.242$ \\
\hline 60 & 0 & 1.741 & 0 & 0.0076 & 0.5683 & $0.268: 0.263: 0.467$ & $0.003: 0.992: 0.004$ \\
\hline 70 & 0 & 0 & 1.434 & 0.0295 & 0.0927 & $0.690: 0.080: 0.229$ & $0.202: 0.023: 0.774$ \\
\hline 100 & 7.339 & 0 & 0 & 0.0023 & 0.3063 & $0.037: 0.230: 0.733$ & $0.968: 0.012: 0.020$ \\
\hline 110 & 0 & 2.048 & 0 & 0.0066 & 0.0017 & $0.034: 0.006: 0.960$ & $0.058: 0.335: 0.607$ \\
\hline 160 & 0 & -1.643 & 0 & 0.0006 & 0.2528 & $0.091: 0.037: 0.872$ & $0.002: 0.997: 0.001$ \\
\hline 170 & 0 & 0 & -1.135 & 0.0004 & 0.1336 & $0.206: 0.040: 0.754$ & $0.001: 0.001: 0.998$ \\
\hline 200 & 9.339 & 0 & 0 & 0.0037 & 0.3246 & $0.030: 0.021: 0.948$ & $0.955: 0.003: 0.042$ \\
\hline 210 & 0 & 1.048 & 0 & 0.0021 & 0.0016 & $0.074: 0.045: 0.880$ & $0.085: 0.483: 0.431$ \\
\hline 220 & 0 & 0 & 1.124 & 0.0009 & 0.0889 & $0.338: 0.584: 0.078$ & $0.003: 0.004: 0.993$ \\
\hline 270 & 0 & 0 & 1.079 & 0.0032 & 0.0532 & $0.413: 0.156: 0.431$ & $0.019: 0.007: 0.974$ \\
\hline 300 & 4.637 & 0 & 0 & 0.0016 & 0.0991 & $0.474: 0.014: 0.512$ & $0.962: 0.002: 0.036$ \\
\hline 310 & 0 & 1.515 & 0 & 0.0014 & 0.2750 & $0.099: 0.014: 0.887$ & $0.0001: 0.998: 0.001$ \\
\hline 320 & 0 & 0 & 0.529 & 0.0037 & 0.0082 & $0.105: 0.007: 0.887$ & $0.044: 0.004: 0.952$ \\
\hline 350 & 9.862 & 0 & 0 & 0.0011 & 0.2852 & $0.122: 0.048: 0.830$ & $0.965: 0.008: 0.026$ \\
\hline 360 & 0 & 1.768 & 0 & 0.0026 & 0.1795 & $0.020: 0.026: 0.954$ & $0.001: 0.9987: 0.002$ \\
\hline 400 & 3.088 & 0 & 0 & 0.0074 & 0.0859 & $0.112: 0.016: 0.872$ & $0.879: 0.007: 0.112$ \\
\hline 410 & 0 & -0.0830 & 0 & 0.0067 & 0.0089 & $0.149: 0.031: 0.820$ & $0.141: 0.080: 0.779$ \\
\hline 420 & 0 & 0 & 0.644 & 0.0040 & 0.0134 & $0.163: 0.068: 0.769$ & $0.043: 0.016: 0.941$ \\
\hline
\end{tabular}

TABLE I: Performance evaluation on Dataset 1

suitability in using them at a particular situation, whereas integrity marker obtained for map data encompasses the effect of map matching process and adequacy of geographic information available from the map. Along with integrity markers, a set of fault predictors are also monitored in the proposed process which acts as a self-assessment marker for the process itself. The novelty of the proposed method lies in the fact that instead of a fault detection and isolation (FDI) framework currently used in integrity monitoring of localization applications, a set of more generalized and handcrafted fluid integrity markers can be used, that can account for a variety of situations without the use of empirical thresholds or rigid logic systems. Hence, instead of bi-state or tri-state fault predictions on each data source, the proposed method provides a weighting scheme for data sources with which a sensor-fusion method can decide how much importance can be given to each data source at any given time.Along with the Fault Predictors for self-assessment of the process, the proposed method is an improvement upon the current stateof-the-art sensor data integrity assessment tools.

\section{FUTURE WORKS}

Different types of road structures, imaging solutions and positioning systems will be included in the further investigations. According to the number and the modality of the sensors used in particular applications, different choices for common model and cross-consistency analysis method can be proposed. The proposed method will be tested in more scenarios generated by driving simulators as well as on realworld data from an experimental vehicle. As mentioned in section $\mathrm{V}$, integrity assessment at source level is a prior step to improve the quality of the sensor-fusion process. The future prospective of this work is in direction of developing a map-aided multi-sensor fusion method that can take full advantage of this integrity assessment output. Ultimately, a functional localization framework where integrity monitoring is intrinsically embedded into different stages -data acquisition, data association and data fusion- is expected to be developed in this work.

\section{REFERENCES}

[1] Federal Radionavigation Plan. US Department of Defense, 2009, ch. 1.

[2] N. R. Velaga, M. A. Quddus, A. L. Bristow, and Y. Zheng, "Mapaided integrity monitoring of a land vehicle navigation system," IEEE Transactions on Intelligent Transportation Systems, vol. 13, no. 2, pp. $848-858,2012$

[3] O. Le Marchand, P. Bonnifait, J. Ibañez-Guzmán, and D. Betaille, "Automotive localization integrity using proprioceptive and pseudo-ranges measurements," in Accurate Localization for Land Transportation, ser. Les Collections de l'INRETS, vol. 125, Paris, France, Jun. 2009, pp. $7-12$.

[4] M. Worner, F. Schuster, F. Dolitzscher, C. G. Keller, M. Haueis, and K. Dietmayer, "Integrity for autonomous driving: A survey," in 2016 IEEE/ION Position, Location and Navigation Symposium (PLANS), April 2016, pp. 666-671.

[5] C. Zinoune, P. Bonnifait, and J. Ibañez-Guzmán, "Integrity monitoring of navigation systems using repetitive journeys," in 2014 IEEE Intelligent Vehicles Symposium Proceedings. IEEE, 2014, pp. 274-280.

[6] J. Palmqvist, "Integrity monitoring of integrated satellite/inertial navigation systems using the likelihood ratio," 1996.

[7] Y. Yang and J. Xu, "Gnss receiver autonomous integrity monitoring (raim) algorithm based on robust estimation," Geodesy and Geodynamics, vol. 7, no. 2, pp. $117-123,2016$.

[8] L. Li, M. Quddus, and L. Zhao, "High accuracy tightly-coupled integrity monitoring algorithm for map-matching," Transportation Research Part C: Emerging Technologies, vol. 36, pp. 13-26, 2013.

[9] C. Zinoune, P. Bonnifait, and J. Ibañez-Guzmán, "Sequential fdia for autonomous integrity monitoring of navigation maps on board vehicles," IEEE Transactions on Intelligent Transportation Systems, vol. 17, no. 1, pp. 143-155, 2016.

[10] J. E. Boritz, "Is practitioners' views on core concepts of information integrity," International Journal of Accounting Information Systems, vol. 6 , no. 4 , pp. $260-279,2005$.

[11] R. Toledo-Moreo, M. A. Zamora-Izquierdo, B. Ubeda-Minarro, and A. F. Gomez-Skarmeta, "High-integrity imm-ekf-based road vehicle navigation with low-cost gps/sbas/ins," IEEE Transactions on Intelligent Transportation Systems, vol. 8, no. 3, pp. 491-511, Sep. 2007.

[12] A. Geiger, P. Lenz, C. Stiller, and R. Urtasun, "Vision meets robotics: The kitti dataset," The International Journal of Robotics Research, vol. 32, no. 11, pp. 1231-1237, 2013. 